\title{
A Survey on Efficient Power Consumption Method for Continuous Location-Based Spatial Queries in Mobile Environment
}

\author{
Vijay Kumar and K. John Singh* \\ School of Information Technology and Engineering, VIT University, Vellore, Tamil Nadu, India
}

Received 5 February 2014; Accepted 29 July 2014

\begin{abstract}
In today's growing world saving of time and energy is much considerable. Mobile users are very common for human beings. It is beneficial in use not only for call but also for different uses i.e. find a particular place in unknown city or place. It saves both time and energy towards searching the place. Many researchers have been done in this regard. But they have problem like consuming time and speed to search the location by mobile. Approach: This paper proposed algorithm based on circular location finder (CLF). There are many algorithms available like proxy based location search for continuous near neighbor $(\mathrm{CNN})$, estimated valid region (EVR), and estimated window vector (EWV) for region search. These are not efficient in sense of consumption of time and energy. Results: Based on our study, circular location finder (CLF) increases approximately $68 \%$ speed and decrease 3 times power consumption taken by mobile application. CLF algorithm is efficient in both speed and power consumption
\end{abstract}

Keywords: mobile computing, spatial query processing, location based services, windows frame.

\section{Introduction}

The revolution of cyber world is challenging as well as comforting. Nowadays, people are offering mobile enabled services because it is portable and easy to carry at anywhere. Because it helps people in many ways like voice chat, video chat, gaming, web surfing for entertainment etc. In same concern person visit to unknown place then it need to be hotel for stay and food. But major problem is that how to get it these place. Either search by self to ask local people or reach with help of auto/rickshaw. Anyway it is not efficient solution of this problem because hotel where you reach with help of other people may be so far instead there are good hotels available in near region. So this paper tries to avoid this problem not only nearest location find but also quick response for query.

In circular location finder algorithm distance will be increase continuously according to time slot. It is able to reduce the power or energy of system. Distance will be major in form of radius. Figures 1 illustrate the step by step process of circular location finder.

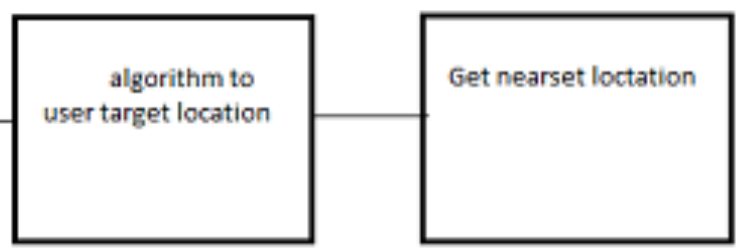

Fig. 1. Process for find circular location

Proxy server set the current location of mobile according to global locality and it uses data structure techniques for finding nearest node/location by calculating using algorithm like shortest path, minimum spanning tree. Then make the comparison based on the result of these algorithms. Here distance will be increase in spatial queries.

Spatial query using same point will increase the speed and decrease the time for search the nearest location according to need.

\footnotetext{
*E-mail address: johnsinghaj@yahoo.com

ISSN: 1791-2377 @ 2014 Kavala Institute of Technology. All rights reserved.
}

\section{Materials and Methods:}

As already mentioned, location search algorithms namely, EVR, EWV based on data structure techniques be proposed. The key concept and principle of these algorithms are discussed in this section. And newly proposed system also describe in a way that can efficiently overcome the drawbacks of existing technologies like speed, power consumption, CPU uses etc.

Proxy based approach to continuous NN: In proxy based approach take valid region of spatial queries at mobile is effective to reduce query load at server [1]. D. Lee, B. Zheng, and W.-C. Lee, "Data Management in LocationDependent Information Services," IEEE Pervasive Computing (2005), The proxy creates the estimate valid 
region by exploiting spatial and temporal locality. There are also proposed NN queries for estimate valid region using minimum use of memory cache.

B. Zhen, "Cache Invalidation and Replacement Strategies for Location-Dependent Data in Mobile,"(2009),For make query more near estimated use windows frame vector [3]. It will accelerate the estimation power of valid region using window based query.

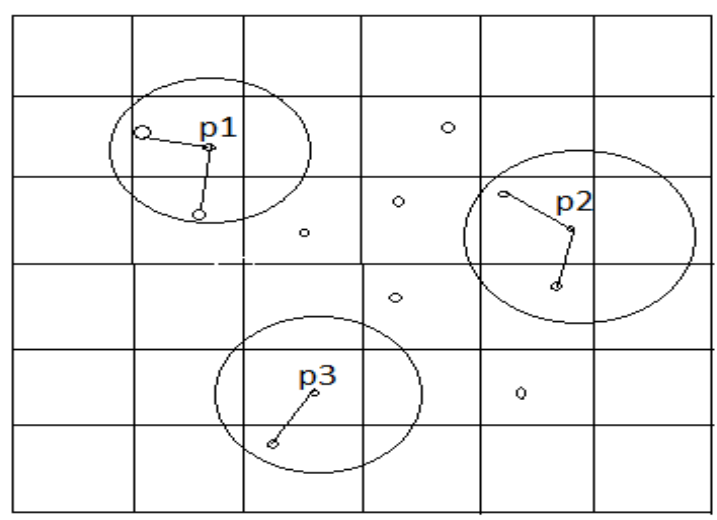

Fig: 2 EVR for NN

In figure 2 many spatial regions are crated for find nearest region. It will work efficiently for minimum frame but when frame will increase it will leave the valid region without calculated as shown in above figure. Region outside the circle are not mentioned as nearest region while they are looking same or have to be valid region. But it does not cover full area for nearest neighbor.

Nearest Neighbor. So it is the major problem in this algorithm.

Windows estimated vectors: S. Prabhakar, Y. Xia, D.V. Kalashnikov "Query Indexing and Velocity Constrained Indexing(2008) This algorithm is introduced for minimize power consumption and reduce the load of server; it is much batter estimate the valid region as compare previous algorithm[ 2]. Y. Cai, K.A. Hua, and G. Cao, "Processing Range-Monitoring Queries on Heterogeneous Mobile Objects."(2009) But it is not much effective in case of speed and time. In figure 3 shows the direction of

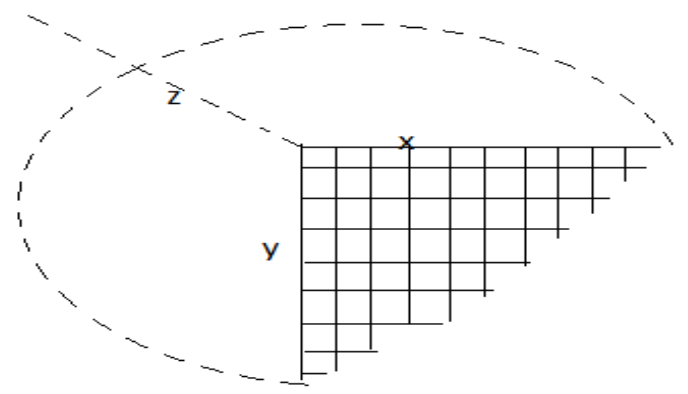

Fig: 3. Vector frame

Circular location finder algorithm: It is based on data structure shortest path algorithm. It finds the location of place unlike the previous techniques. K. Mouratidis, D. Papadias, S. Bakiras "A Threshold- Based Algorithm for
Cont. Monitoring of k Nearest Neighbors,"(2009). It will much consider about speed and quick response of query. It finds the location in circle as shown in figure 4. First it look for nearest neighbor in particular distance than it accelerate the distance and again measure the shortest path from latest shortest point and rapidly grow in same way [4]. It will reduce the spatial query time and reply fast.

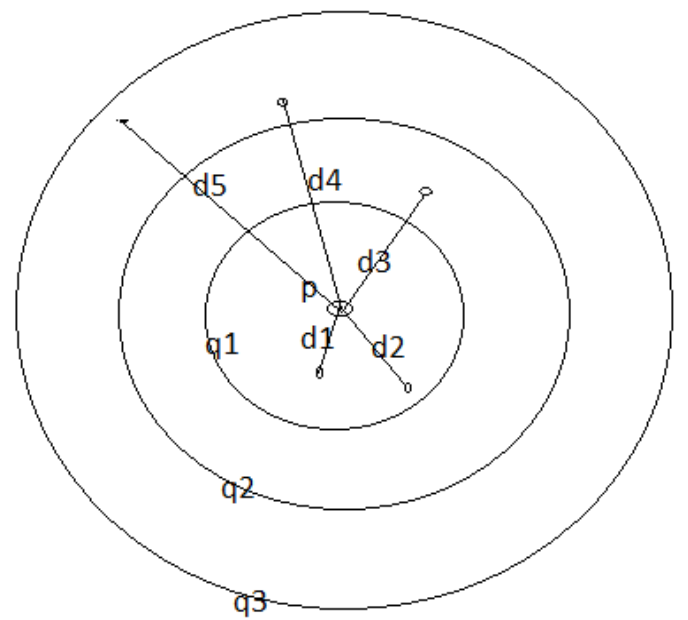

Fig: 4 Circular nearest neighbor

\section{Results}

Performance of EVR for NN: It create discrete region for estimate valid region but not cover all region they might be valid. Because it use discrete region so small cache can be used efficiently. It increases cache uses efficiency up to $20 \%$.

Performance of WEV: It uses window frame and index for estimate much valid region. So it is more efficient power consumption for valid region in mobile environment. It uses power consumption up to $35 \%$.

Performance of Circular based location finder: It find the location in circular format and covers up to $60 \%$ much region than previous algorithm. Its reason is that circle covers more area than square format. If more area considered than definitely it will cover much nearest neighbor.

Graphically comparison between EVR for NN and Circular based techniques:

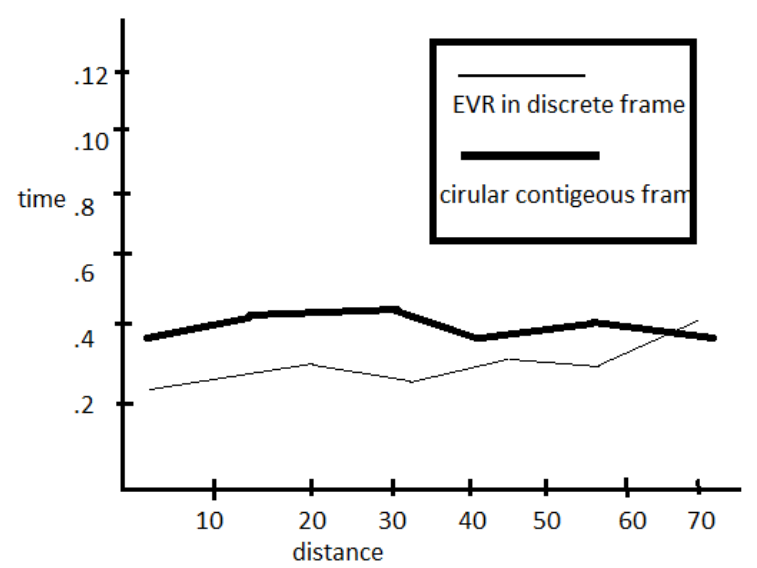

Fig: 5. Graphical representations 4. Conclusion. 
Here many data structure based approaches are used to estimate valid region for nearest neighbor. But circular based approach much suitable for quick response because it always accelerating its length to find next nearest neighbor. And cover much area in short period of time.

\section{References}

1. D. Lee, B. Zheng, and W.-C. Lee, "Data Management in LocationDependent Information Services," IEEE Pervasive Computing,vol. 1, no. 3, pp. 65-72, July-Sept. 2007.

2. B. Zheng, J. Xu, and D.L. Lee, "Cache Invalidation and Replacement Strategies for Location-Dependent Data in Mobile Environments," IEEE Trans. Computers, vol. 15, no. 10, pp. 1141- 1153, Oct. 2008.

3. X. Gao, J. Sustersic, and A.R. Hurson, "Window Query Processing with Proxy Cache," Proc. Seventh IEEE Int'l Conf. Mobile Data Management, 2006.

4. S. Prabhakar, Y. Xia, D.V. Kalashnikov, W.G. Aref, and S.E.Hambrusch, "Query Indexing and Velocity Constrained Indexing: Scalable Techniques for Continuous Queries on Moving Objects," IEEE Trans. Computers, vol. 51, no. 10, pp. 1124-1140, Oct. 2007

5. [B. Gedik and L. Liu, "Mobieyes: A Distributed Location Monitoring Service Using Moving Location Queries," IEEE Trans.Mobile Computing, vol. 5, no. 6, pp. 1384-1042, Oct. 2006.

6. X. Xiong, M.F. Mokbel, and W.G. Aref, "Sea-Cnn: Scalable Processing of Continuous k-Nearest Neighbor Queries in SpatioTemporal Databases," Proc. IEEE Int'l Conf. Data Eng., pp. 643654, 2008.

7. K. Mouratidis, D. Papadias, S. Bakiras, and Y. Tao, "A ThresholdBased Algorithm for Continuous Monitoring of $\mathrm{k}$ Nearest Neighbors," IEEE Trans. Knowledge Data Eng., vol. 17, no. 10,pp. 1451-1464, Nov. 2009. 